\title{
Synchronous Surface Pressure and Velocity Measurements of standard model in hypersonic flow
}

\author{
Sun Zhijun ${ }^{1}$, GU Yunsong ${ }^{1}$, CHENG Keming ${ }^{1}$, Di peng ${ }^{2}$, Yingzheng Liu ${ }^{2}$ \\ ${ }^{1}$ College of Aerospace Engineering, Nanjing University of Aeronautics and Astronautics(NUAA), Nanjing , China \\ ${ }^{2}$ Gas Turbine Research Institute/School of Mechanical Engineering, Shanghai Jiao Tong University, Shanghai, China
}

\begin{abstract}
Experiments in the Hypersonic Wind tunnel of NUAA(NHW) present synchronous measurements of bow shockwave and surface pressure of a standard blunt rotary model (AGARD HB-2), which was carried out in order to measure the Mach-5-flow above a blunt body by PIV (Particle Image Velocimetry) as well as unsteady pressure around the rotary body. Titanium dioxide $\left(\mathrm{Al}_{2} \mathrm{O}_{3}\right)$ Nano particles were seeded into the flow by a tailor-made container. With meticulous care designed optical path, the laser was guided into the vacuum experimental section. The transient pressure was obtained around model by using fast-responding pressure-sensitive paint (PSP)sprayed on the model. All the experimental facilities were controlled by Series Pulse Generator to ensure that the data was time related. The PIV measurements of velocities in front of the detached bow shock agreed very well with the calculated value, with less than 3\% difference compared to Pitot-pressure recordings. The velocity gradient contour described in accord with the detached bow shock that showed on schlieren. The PSP results presented good agreement with the reference data from previous studies. Our work involving studies of synchronous shock-wave and pressure measurements proved to be encouraging.
\end{abstract}

\section{Introduction}

The development of optical measurement technology shows the potential advantages in the research of flow field measurement. Since the advent of Laser Doppler Velocimetry (LDV), Optical measurement techniques have been applied and developed for a long time in the aerodynamic experiments. The Particle Image Velocimetry (Particle Image Velocimetry, PIV) highly desirable for capturing the flow structures due to their low intrusiveness and high spatial resolution, gradually becoming predominant technology in flow field measurements ${ }^{[1-4]}$

By using the method of laser speckle to capture the image of the particle displacement at a certain time interval, the velocity of particle motion was obtained by the cross-correlation method. The particles are distributed uniformly in the flow field, along with the fluid, so that the velocity of the particles can indicate the velocity of the current fluid. There for, the following properties of particles and the capture of the particle image become the key factors in the accuracy of PIV measurements.

In recent years, with the advent of high-energy lasers and high resolution digital image recorders, the PIV technology made supersonic flow field measurement possible. But for hypersonic flow, the high speed, high gradient and compressibility bring a lot of difficulties to the application of PIV in hypersonic flow field measurement.
The hypersonic flow field has a large velocity gradient, especially around the shock wave, which has a strong deceleration effect on the shock wave surface. Due to the effect of inertia, the particles in flow field cannot remain completely follow, the precision of velocity measurement has a great influence ${ }^{[5,6]}$. Flow compressibility introduces two difficulties, namely the large spatial variation of the particle density and the inhomogeneous refractive index throughout the flow field, results in a distortion of laser propagation. ${ }^{[7]}$

As a global pressure testing technology, PSP (Pressure-Sensitive Paint) has been widely used in wind tunnel test. ${ }^{[8]}$ However, PSP measurement in hypersonic flows is usually challenged by the paint response time. The fast-responding PSP typically have frequency response of a few hundred $\mathrm{Hz}$ which is sufficient for steady measurement ${ }^{[9-11]}$.

With the continuous improvement of wind tunnel measurement and control technology, the problem in flow was becoming more and more complicated. For flow field research, a variety of testing methods were often required. Synchronous measurement technology can be used to simultaneously obtain test content (e.g. the aerodynamic force, surface pressure) in a single test. ${ }^{[12,13]}$ The test results obtained by this method are relevant in time and make up for the defects of independent measurement technology in time synchronization, which is helpful for the study of the unsteady aerodynamic problems. On the other hand, the synchronous 
measurement can reduce the number of tests and improve the economy of single wind tunnel test.

The current study focused on overcoming the challenges in simultaneous shock-wave and pressure measurements in transient hypersonic flows by using optical technology. The combined PIV/PSP measurement was applied in synchronous test on the AGARD hb-2 standard model at $\mathrm{Ma}=5$.

\section{Experiment setup}

\subsection{Hypersonic facility}

The experiment was conducted in a conventional hypersonic blow-down facility $\left(\mathrm{NHW}^{[14]}\right)$ at Nanjing University of Aeronautics and Astronautics. As shown in Fig. 1, NHW is an intermittent blow-down wind tunnel with vacuum suction to produce hypersonic flow. This tunnel mainly consists of a high-pressure gas source, a heater, a stabilizing section (with nozzle), a vacuum test section, a diffuser, a vacuum system and an electrical control system. A support mechanism with variable angle of attack is set in the test section. Four sets of axisymmetric supersonic nozzles with nominal Mach number of 5, 6, 7 and 8 are available.

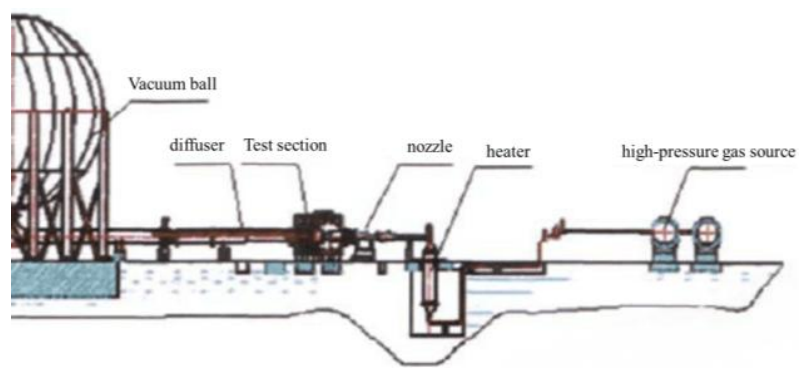

Figure 1. Sketch of Nan-hang Hypersonic Wind tunnel (NHW)

The tunnel has a $2 \mathrm{~m} \times 2 \mathrm{~m} \times 2.5 \mathrm{~m}$ rectangular plenum chamber. The front and back ends of the plenum chamber are connected to the nozzle and the vacuum respectively. A pair of observation windows $(\Phi 400 \mathrm{~mm})$ is located on both side walls of the test section, which has been used to monitor the shock wave structures around the model. On top of the experimental section has a $0.6 \times 0.6 \mathrm{~m} 2$ optical observation window with germanium glass, used in optical measuring system. A free jet - type test section $(\Phi 500 \mathrm{~mm})$ was installed inside the plenum chamber. The model support mechanism can accurately provide the Angle of attack required during the test.

The typical operating parameters of this wind tunnel at $\mathrm{Ma}=5$ are listed in Table 1 . The wind tunnel is equipped with a $300-\mathrm{mm}$-diameter color Schlieren system for flow visualization, which was used to provide the flow structures around the nose of the model in the current study.
Table 1. Operating parameters of hypersonic wind tunnel

\begin{tabular}{cc}
\hline Mach number & 5 \\
\hline Atmosphere pressure & $101325 \mathrm{~Pa}$ \\
Total pressure/static pressure & 530 \\
Dynamic pressure coefficient & 0.03177 \\
Total pressure & $\sim 500 \mathrm{kPa}$ \\
Total temperature & $\sim 500 \mathrm{~K}$ \\
Static pressure in test section & $\sim 950 \mathrm{~Pa}$ \\
Dynamic pressure in test section & $\sim 16000 \mathrm{~Pa}$ \\
\hline
\end{tabular}

\subsection{AGARD HB-2 test model}

An AGARD HB-2(Hypervelocity ballistic model 2) standard model was selected for the current experimental study, which is a widely used aerodynamic force\& moment measurement model in hypersonic experiments, and abundant wind tunnel test data are available from previous research for validation ${ }^{[15]}$.

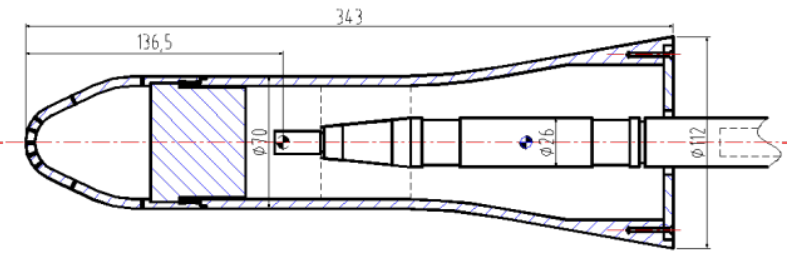

Figure 2. Schematic of AGARD HB-2 model

The test model got a shape similar to a reentry-body, at 0 Angle of attack, the flow field has a symmetrical structure. To prevent the surface from having intense laser reflection, the blackening treatment was obtained on the surface of test model, which reduces the reflection and improves the particle image signal to noise ratio (figure 3).
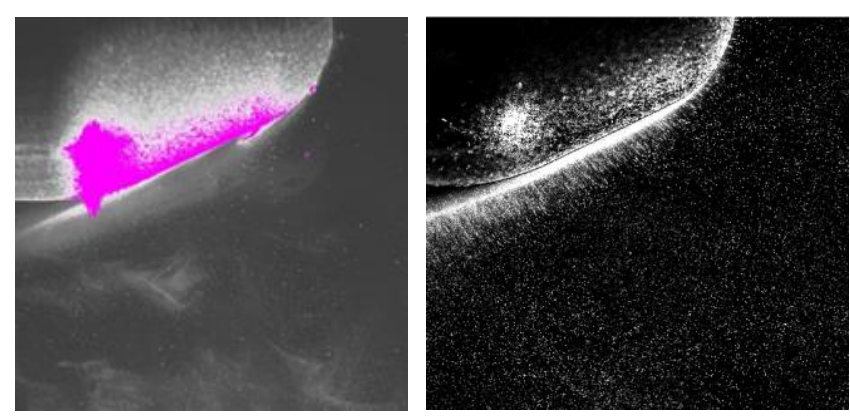

Figure 3. Particle image in the test area (left: baseline, right: model surface blackening treatment) 


\subsection{Pressure sensitive measurement techniques}

The surface pressure was measured by PSP, which consists of a polymer-ceramic (PC) binder and four (pentafluorophenyl) porphyrin platinum (PtTFPP) as pressure sensor. The PC binder is composed of high concentration ceramic particles with a small amount of polymer to physically hold the ceramic particles to the surface ${ }^{[16]}$. The binder formed porous surface, which can interact immediately with oxygen, resulting in a highfrequency response.

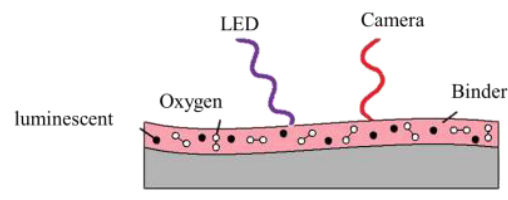

Figure 4. Technical principle of Fast-responding $\operatorname{PSP}^{[8]}$

Several pressure reference taps are seted on the surface of the model to calibrate the PSP measurement results(Fig.5).The pressure reference taps uses Kulite sensor for dynamic measurement, and the stagnation pressure reference tap is set at the head of the model to measure the change of head stagnation pressure during the whole test process.

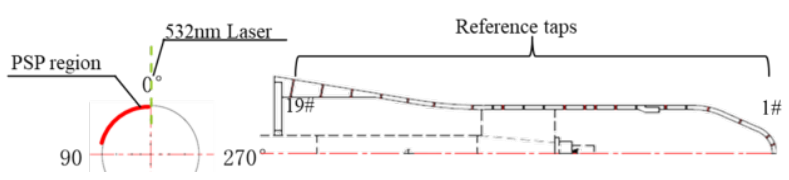

Figure 5. Paints and reference taps on the AGARD HB-2 model(left: PSP and PIV laser position, right: reference taps position on the model)

The experimental setup for the wind tunnel experiments is shown in Fig. 6. The HB-2 model coated with PSP was placed in the test section on the support system. In order to achieve the synchronization measurement of PIV and PSP, the experimental optical path was carefully designed. In order to meet the complex test conditions, the fluidized bed form of particle generator and the injection orifice upstream of the nozzle are designed. The solid nanoparticles are injected into the mainstream by the particle generator, which flows through the expansion of the nozzle.

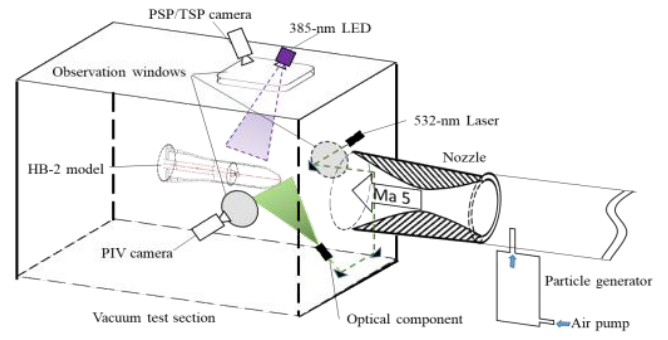

Figure 6. Schematic of the particle seeding device and optical path in the NHW wind tunnel.
The laser beam passes through the observation window, and the mirror group reaches the bottom of the model. A set of lenses turns the beam of light into a piece of light, shining on the symmetrical surface of the model. The PIV camera with a $200 \mathrm{~mm}$ lens and a $532 \pm 5 \mathrm{~nm}$ filter is set in another observation window for shooting, which provide 2048 by 2048 pixels images in a $75 \times$ $75 \mathrm{~mm} 2$ area. Continuous illumination was provided by the 385-nm LED through a glass window on the top of the test section. The luminescent signal was recorded continuously by a 12-bit high-speed camera at $500 \mathrm{~Hz}$ through a 570-nm long wave pass filter.

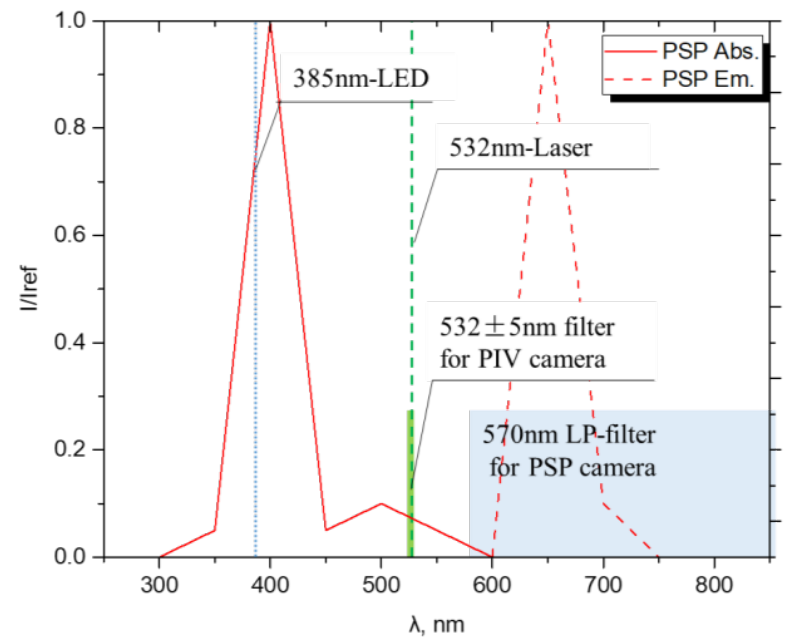

Figure 7. Absorption and emission of PSP (filter for PSP\&PIV camera are shown with filled blocks)

\subsection{Data acquisition}

The PIV and PSP data were recorded simultaneously for a period of about 20 s during each wind tunnel running. The external trigger signal for data collection is given by the synchronizer. When the wind tunnel starts working, the initial trigger signal is provided. The synchronizer sends a collection signal simultaneously to each collector after receiving the initial trigger signal (Fig 8). Single pulse triggering for particles generator, $2 \mathrm{~Hz}$ square signal for PIV camera, $500 \mathrm{~Hz}$ square signal for PSP camera. All the control signals are started in the same time and recorded by the National Instruments data acquisition (NI DAQ) system.

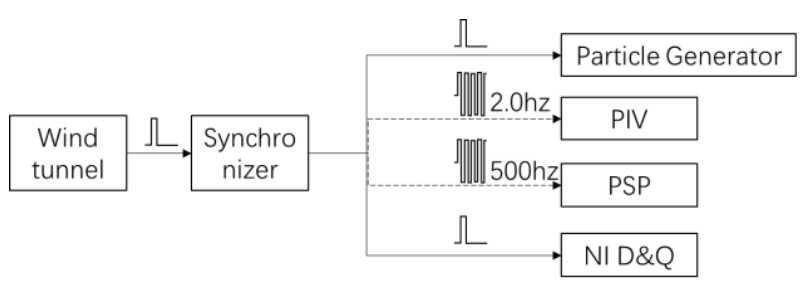

Figure 8. Schematic of system control links 


\section{Results and discussion}

\subsection{Flow structures}

In the case of $\mathrm{Ma}=5$, the flow velocity needs to be calculated by the isentropic relation of the compressible flow. The formula is as follows:

$$
V_{\infty}=a \cdot M a=\sqrt{\gamma R T} \cdot M a=M a \cdot \sqrt{\frac{\gamma R T_{0}}{1+\frac{\gamma-1}{2} M a^{2}}}
$$

During the experiment, $\mathrm{T}_{0}$ (Total temperature) was about $500 \mathrm{~K}$, and $\gamma=1.4, \mathrm{R}=287.05$. So the flow velocity can theoretically be calculated as $\mathrm{V}_{\infty}=915.0 \mathrm{~m} / \mathrm{s}$.

Figure 9 is the result of the velocity distribution of the head section after the test flow field is established. The measured velocity in front of the head section is about $900.3 \mathrm{~m} / \mathrm{s}$, which is consistent with the theoretical value of the calculation.
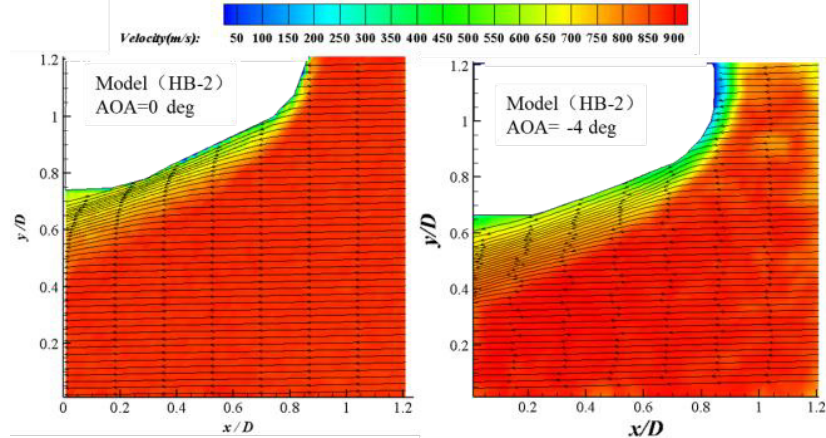

Figure 9. PIV result of velocity field around the dead area of AGARD HB-2 model(The average of 20 images in the steady stage of flow field)

The velocity gradient of the head area is shown in figure 10 . The head section of the model is enveloped by an arched slows down area, which extends and gradually moves away from the model in downstream direction. The gradient is larger in the middle area near the symmetrical surface, and gradually decrease in downstream direction. According to the distribution of velocity gradient, it is easy to see that there is a bow shock wave that surrounds the head of the model.

The color Schlieren images recorded at $\mathrm{Ma}=5$ are shown in Fig. 11 for $\alpha=-4^{\circ}$. The spatial position of the bow shock in schlieren images were well matched with PIV measurements.

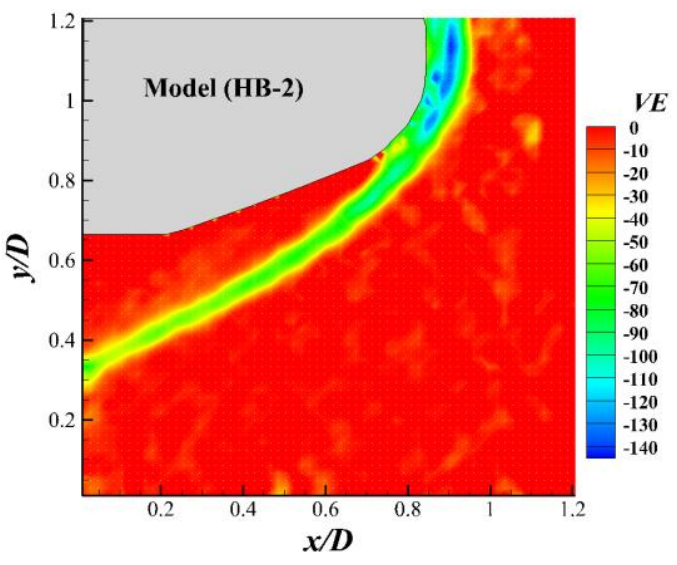

Figure 10. Result of velocity gradient $(\mathrm{AOA}=-4)$

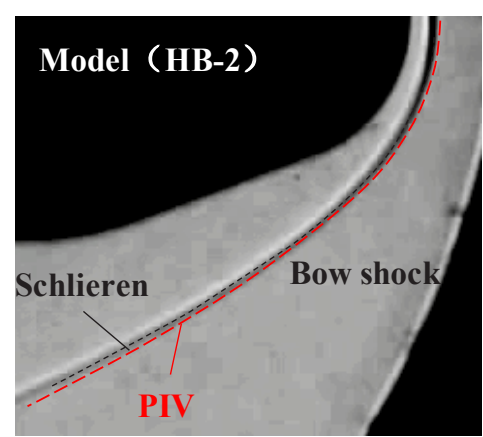

Figure 11. PIV result of bow shock wave (color schlieren taken at $\mathrm{AOA}=-4$ )

\subsection{Surface Pressure}

The surface pressure field of the cylindrical section measured by PSP is presented in Fig. 12. Due to the high surface shear stress and particles in hypersonic flow, the PC binder coating on the cone section surface was destroyed. Note that PSP data were not available on most of destroyed area.

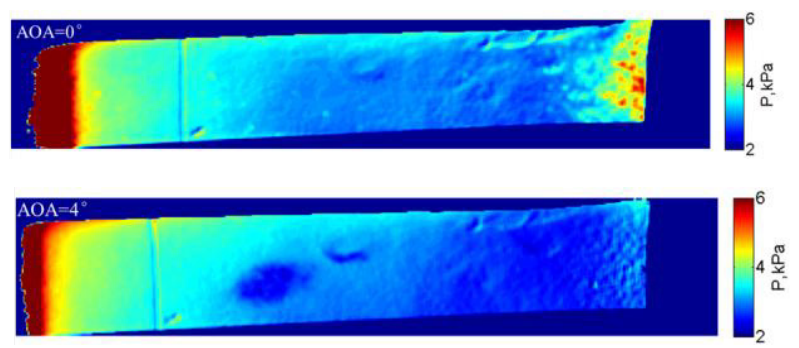

Figure 12. Time-averaged surface pressure distribution of the straight section during the steady stage of flow field(up: at $\mathrm{AOA}=0$ deg, down: at $\mathrm{AOA}=-4 \mathrm{deg}$ ) 
The time-averaged pressure field on the generatrix of the model measured by PSP has been normalized by the stagnation pressure ( $\mathrm{P} 0$ from reference tap-1\#) and is presented in Fig.5. The hollow triangle symbol in the diagram represents the pressure data of the reference taps(in Fig.13), measured by Kulite absolute pressure transducer.

The streamwise pressure profile is compared well with data obtained by pressure taps at the same Mach number in DAL(Douglas Aero-physics Laboratory ${ }^{[15]}$ ), as shown in Fig. 14. The pressure falls suddenly at the junction of cylindrical and cone sections due to an obvious change in the model profile. Then it decreases gradually and reaches a minimum between $\mathrm{x} / \mathrm{L}=0.5$ and 0.6 , finally climbs up at the start of the tail section. Noted that the measurement error between PSP and reference tap is about $0.2 \%(400 \mathrm{~Pa})$, this error may have been caused by the following factors:(1) sensor paint inhomogeneity of PSP which results in accidental error, (2) system error due to air flow heating effect, (3) the uneven distribute of air density caused by shock waves in the flow filed resulted in optical measurement error.

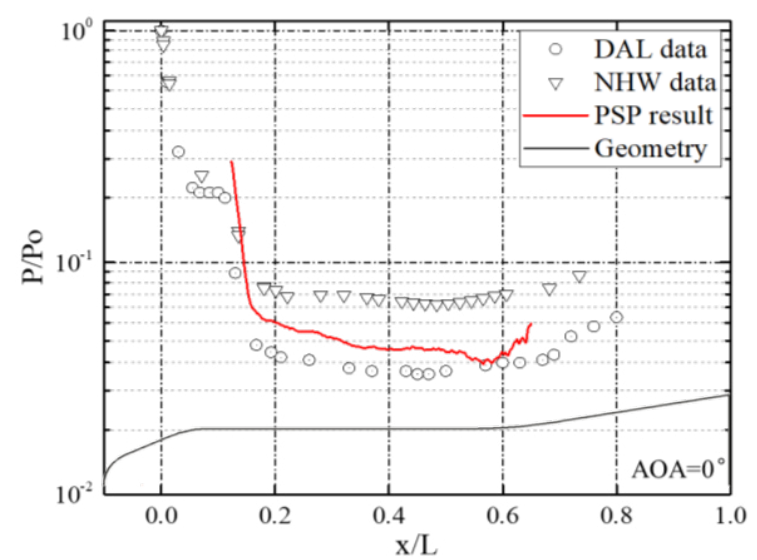

Figure 13. Results of surface pressure profile compared with previous data from DAL

\section{Conclusions}

Simultaneous PIV and PSP measurements in hypersonic flows are quite challenging mostly due to complex flow conditions. In the current work, the spatial position of the bow shock is accurate captured with PIV measurements. The measurements of the PSP are also consistent with previous studies, the evolutions of pressure during the test period were clearly visualized.

The combined PIV/PSP measurement technique has shown great potential for synchronous shock-wave and surface pressure measurements in hypersonic flows.

\section{Acknowledgement}

The author would like to thank all members of Innovation Laboratory of Flight Measurement and Flow Control of NUAA for the interesting and fruitful discussions during the course for this cooperation.

\section{Funding}

The authors disclosed receipt of the following support for the research, authorship, and publication of this article : The research work was supported by the National Science Foundation of China (No.11672134).

\section{References}

1. Westerweel J, Elsinga G E, Adrian R J. Annual Review of Fluid Mechanics, 2013, 45(45):409.

2. Adrian $\mathrm{R}$ J.Experiments in Fluids ,2005 , 39(2):159-169

3. Stanislas M, Kompenhans J, Westerweel J: Particle Image Velocimetry (Springer Berlin Heidelberg, 2007).

4. Fulvio Scarano . Topics in Applied Physics, 2008, 445-463.

5. Melling A. Measurement Science \& Technology, 1999, 8(12):1406-1416.

6. Schrijer F F J,Scarano.7th International Symposium on Particle Image Velocimetry. 2007

7. Elsinga G E, Oudheusden B W V, Scarano F. Experiments in Fluids, 2005, 39(2):246-256.

8. Liu T, Sullivan J P:Pressure- and TemperatureSensitive Paints(Springer Berlin Heidelberg, 2005).

9. Gregory J W, Sakaue H, Liu T, et al. Annual Review of Fluid Mechanics, 2014, 46(1):303-330.

10. Xiang X, Yuan M, Yu J, et al. Aiaa Aerodynamic Measurement Technology and Ground Testing Conference. 2013.

11. Okabe T, Miyazaki T, Sakaue H. Aerodynamic Measurement Technology, Ground Testing, and Flight Testing Conference. 2013:2201-2204 Vol.2.

12. Klein C: Recent Developments of PSP and TSP at DLR for Application to Transonic Flows in Industrial Wind Tunnels( Nein, 2008).

13. Egami Y, Fey U, Klein C, et al. Measurement Science \& Technology, 2012, 23(11):286-286.

14. XU X, CHENG K.M, WANG. Journal of Experiments in Fluid Mechanics (2009)

15. Gray, J. D. Summary report on aerodynamic characteristics of standard models HB-1 and HB-2. Aedc-tdr-64-137, July 1964.

16. Peng, Di, et al. Experiments in Fluids.57.12(2016). 